\title{
O PAPEL DA HARMONIZAÇÃO OROFACIAL EM PACIENTES SEQUELADOS POR PARALISIA PARCIAL PERIFÉRICA - RELATO DE CASO CLÍNICO
}

\author{
Letícia do Valle Reis
}

RESUMO: O papel da Harmonização Orofacial em pacientes sequelados por Paralisia Parcial Periférica é de grande importância no sentido de se reestabelecer um convívio social adequado e elevar a auto estima da pessoa comprometida. O trabalho apresenta um caso clínico em que se conseguiu, através do uso da toxina botulínica, laserterapia e administração de vitaminas do complexo $\mathrm{B}$, trazer de volta a harmonia facial do paciente, perdida desde o acometimento da paralisia, há oito anos.

Palavras-chave: Harmonização orofacial. Paralisia, Toxina botulínica. Laserterapia.

ABSTRACT: The role of Orofacial Harmonization in patients affected by Peripheral Facial Paralysis is very important to reestablish the affected person's social life and self-esteem. This paper presents a clinical case where it was possible, using botulinum toxin, laser therapy and administration of vitamins of $\mathrm{B}$ complex group, to bring back the facial harmony of a patient, which had been lost since the beginning of the paralysis, eight years ago.

Keywords: Orofacial harmonization. Paralysis. Botulinun toxin. Laser therapy.

\section{INTRODUÇÃO}

Descrita pela primeira vez em i82ı pelo britânico Sir Charles Bell, a Paralisia de Bell consiste de acometimento do sétimo nervo craniano de forma aguda. Ela não possui causa detectável, ocorre unilateralmente e não apresenta causa específica. Sua etiologia pode estar associada a trauma, compressão, infecção, inflamação ou ser de origem metabólica, podendo ser precedida por dor na região retro auricular, que persiste por alguns dias e usualmente requer analgesia (FALAVIGNA; et al, 2008).

\footnotetext{
I Dentista especializada em harmonização ortofacial, atuando em estética facial. E-mail: leticiadovallereis.1dvr@gmail.com.
} 
Falavigna, et al (2008) comenta que, em metade desses pacientes, a dor aparece dois a três dias antes da paralisia e nos demais no momento de sua instalação. A paralisia de Bell corresponde de $60 \%$ a $75 \%$ de todos os casos de paralisia facial.

A diminuição da sensibilidade gustativa e da produção de lágrimas é observada em $30 \%$ e $5 \%$ dos casos, respectivamente. Esses sintomas são decorrentes da disfunção parassimpática, pela lesão do nervo intermédio de Wrisberg após sua sinapse com o gânglio geniculano (FALAVIGNA; et al, 2008).

Segundo Falavigna, et al (2008), após a paralisia facial aguda as fibras pré ganglionares parassimpáticas que se projetavam para o gânglio submandibular, podem regredir e se conectar ao nervo petroso maior superficial. Essa regeneração pode causar um lacrimejamento após um estímulo salivatório, conhecido com síndrome de Lágrimas de crocodilo. Esse fenômeno pode ser observado em até 70\% dos casos. A fonofobia é observada em $15 \%$ e resulta da paralisia do músculo estapédio, responsável pela movimentação do estribo, o que consequentemente torna ausente o reflexo estapediano. História familiar é observada em $4 \%$ dos pacientes.

O diagnóstico diferencial da Paralisia de Bell ou Periférica é com a paralisia central. A paralisia facial central é decorrente da lesão acima do núcleo do nervo facial, localizado na ponte, a qual causa a paralisia da extremidade inferior da face, contralateral a lesão, pois o núcleo do nervo facial que inerva a hemiface inferior recebe fibras corticonucleares do hemisfério contra lateral (FALAVIGNA; et al, 2008). Sendo assim, a paralisia facial periférica acomete toda a hemiface, já a paralisia central acomete apenas a porção inferior da face.

A sintomatologia máxima ocorre nas 48 a 72 horas e sua gravidade correlaciona-se com a duração da distensão facial, a extensão da recuperação facial e com o comprometimento da qualidade de vida. Porém, alguns pacientes apresentam recuperação incompleta ou, como no caso a ser relatado, não procuram tratamento (ADONI, 2008). 
Valença, et al (2006) estima que a incidência da Paralisia de Bell, seria de 20 a 30 casos por roo mil habitantes, com prevalência ligeiramente maior entre mulheres. É rara antes dos Io anos de idade e sua incidência biomodal com picos na terceira e na quarta décadas de vida, dependendo da distribuição etária da população. Como fatores de risco para a Paralisia de Bell, tem sido relatados: hipertensão arterial, diabetes mélito, gravidez e puerpério e a infecção pelo vírus da herpes tipo I.

Em um primeiro momento o tratamento da Paralisia Facial Periférica engloba uma terapêutica farmacológica, a reeducação, os métodos físicos e até mesmo cirurgia. Porém alguns pacientes apresentam recuperação incompleta e desenvolvem hipertonia, hipercinesia ou sincinesia, que é apontado como inerentes a regeneração neural, porque a recuperação das fibras ocorre de forma supra numerária, desviada com falhas na transmissão entre axônios adjacentes ou hiperexcitabilidade nuclear (MATOS, 20II).

No entanto, a literatura relacionada à reabilitação orofacial na Paralisia Facial ainda é restrita. $\mathrm{O}$ conhecimento básico da neurofisiologia muscular permite compreender mais os fenômenos da denervação e atrofia. Esse conhecimento é importante para definir técnicas de atuação e eventualmente desenvolver novas estratégias de atuação na reabilitação facial. Contudo, sabemos que a expressão facial é importante para a comunicação e o auto reconhecimento e fundamental para o senso de identificação e habilidade de integração social, uma vez que a face é a primeira forma de reconhecimento do ser humano. Por consequência o indivíduo pode acarretar uma variedade de problemas psicossociais, como depressão, ansiedade, rejeição e até mesmo paranoia.

O objetivo do trabalho foi restabelecer a harmonia facial do paciente, à partir dos conhecimentos da fisiologia e farmacologia, fazendo uso da Toxina Botulínica tipo A nos dois lados da face, a fim de prevenir a assimetria facial iatrogênica, o que vem sendo observada em casos que utilizam a aplicação unilateral, em conjunto com sessões de laser terapêutico de baixa frequência no lado acometido, que visa a obtenção de um aumento da amplitude dos potenciais de ação (função nervosa estimulada) e capacidade de aceleração de regeneração das estruturas nervosas. A administração via oral de vitaminas do 
complexo B também foi utilizada no tratamento, sabendo-se que interfere diretamente no metabolismo do sistema nervoso central, como periférico, e sua deficiência pode causar degeneração do axônio ou da bainha de mielina das fibras nervosas, sendo porém revertido o quadro a partir de seu uso.

\section{OBJETIVO}

Este trabalho propõe o relato de caso clínico de aplicação de toxina botulínica tipo A em ambos os lados da face, laserterapia de baixa frequência no lado acometido da face e uso de vitamina do complexo B em um paciente acometido por paralisia de Bell há 8 anos sem nenhum tratamento, já apresentando sequela.

Os objetivos principais foram ajudar o paciente a restabelecer a harmonia facial o mais breve possível, a fim de que ele voltasse a ter um convívio social sem constrangimento, pois sabe-se que a paralisia facial afeta negativamente a vida do paciente.

Os objetivos secundários foram avaliar a técnica de aplicação da toxina botulínica, distribuída em ambos os lados da face, observar a eficácia da laserterapia combinada com a vitamina B na reversão de sequelas e proporcionar aos demais colegas o conhecimento desse protocolo elaborado e utilizado pela autora.

\section{REVISÃO DE LITERATURA}

A toxina botulínica tipo $\mathrm{A}$ atua ao nível de terminações nervosas, bloqueando a liberação de acetilcolina nas sinapses através da interferência do metabolismo do cálcio. A fraqueza muscular ocorre na área de aplicação do músculo, causando uma diminuição nas terminações nervosas e deixando o músculo inativo durante o seu efeito.

Segundo Gomez, Vasconcelos e Moraes (1999), a paralisia parcial periférica requer abordagem médica, fisioterapêutica e fonoaudiológica. A fisioterapia é indispensável para o objetivo principal de reestabelecer o tropismo, a força e a função muscular. Os recursos sugeridos pela literatura são: Cinesioterapia, massagem e eletrotermoterapia, confirmados por ensaio clínico aleatório e revisão sistemática. 
A recuperação dos tônus musculares ocorre pelo crescimento de brotamentos axonais nas terminações nervosas que reinervam o músculo, o que justifica a escolha da aplicação bilateral, induzindo o lado paralisado a produzir novos brotamentos axonais.

Segundo Viegas, et al (200I), a Laserterapia de Baixa Potência, ou fotobiomodulação, é uma terapia com fundamentos na interação com os tecidos biológicos, à partir da irradiação por um feixe de luz monocromático, colimado e coerente de um determinado comprimento de onda e da absorção desse comprimento de onda através de cromóforos pelo organismo. Cada comprimento de onda tem absorção específica por um ou mais cromóforos, e é isso que faz a indicação do comprimento de onda a ser usado, com base no efeito desejado.

Os cromóforos são fotorreceptores existentes nas células que absorvem a energia dos fótons emitidos. Com isso, produzem efeitos fotoquímicos, fotobiológico e fotofísico nos tecidos, influenciando na modulação celular. Um dos principais cromóforos é o citocromo C oxidase, uma enzima que está na cadeia respiratória, dentro da mitocôndria e participa no transporte de elétrons.

Oliveira (2006) aponta que as vitaminas do complexo B são participantes fundamentais no metabolismo dos carboidratos, lipídeos e proteínas e atuam de formas diferentes em diversos sistemas enzimáticos, mas sempre participam como coenzimas na ativação de inúmeros processos metabólicos. Assim se explica sua importância no metabolismo do sistema nervoso central e periférico. Sua deficiência pode causar degeneração do axônio ou da bainha de mielina das fibras nervosas. Por serem hidrossolúveis, não são armazenadas no organismo de forma considerável, isso ressalta a importância da complementação no processo de recuperação do tecido nervoso.

\section{RELATO DE CASO CLÍNICO}

O paciente M.A.P.O., de 55 anos, gênero masculino, comparece ao consultório dia 13 de Fevereiro de 2021 para tratamento de Harmonização Orofacial, encaminhado por oftalmologista, pois apresentava espasmos hemifaciais, contrações sinéticas, leve lacrimejamento e extremo desconforto relacionado à sua aparência. 
Durante a anamnese, o paciente relatou ter sofrido uma paralisia facial a 8 anos, sem ter procurado nenhum tipo de tratamento. Relatou que, antes do ocorrido, passou por um grande período de estresse emocional e que, em um determinado dia, teve uma dor de ouvido e que logo em seguida começou a perceber o rosto repuxando. No entanto, não procurou tratamento, pois conhecidos disseram que era "Doença do vento" e que achava que não teria solução.

Após orientação e assinatura do termo de aceite, foi agendado o dia para o início do tratamento. Segundo artigo de vários autores publicado na Revista Brasileira de Cirurgia Plástica (2015), a base padronizada de pontos de aplicação para aplicação da toxina evita assimetria iatrogênica após aplicação. A figura abaixo mostra esse mapa de pontos.

Figura I - Divisão didática da face para otimizar técnica de preenchedores

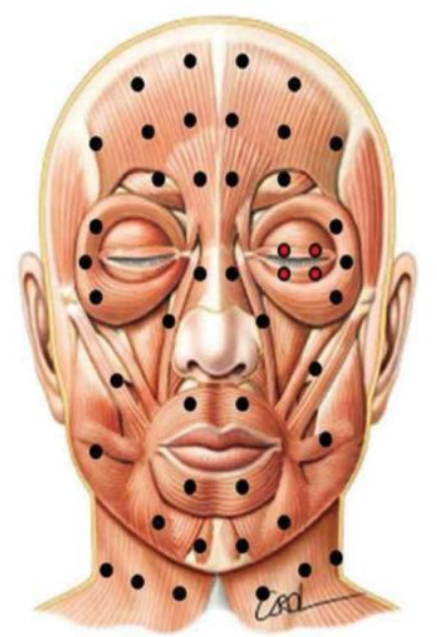

Fonte: Revista Brasileira de Cirurgia Plástica (2015)

A tabela abaixo, elaborada pela autora, relaciona a dose média de UI da Toxina Botulínica tipo A utilizada em cada aplicação, por grupo muscular, em cada lado da face acometido pela paralisia.

Tabela I - Dose média de Toxina Botulínica tipo A por grupo muscular

\begin{tabular}{|c|c|c|}
\hline \multirow{2}{*}{ Grupo Muscular } & Dose média & Dose média \\
contralateral
\end{tabular}




\begin{tabular}{|c|c|c|}
\hline Pré-tarsal & 3,6 & 0,0 \\
\hline Orbicular dos olhos lateral & $6, \mathrm{I}$ & 4,3 \\
\hline Frontal & 2,6 & 2,6 \\
\hline Corrugadores & $\mathrm{I}, 2$ & $\mathrm{I}, \mathrm{O}$ \\
\hline Depressores do lábio inferior & $2, \mathrm{I}$ & $\mathrm{I}, 9$ \\
\hline Elevadores do lábio superior & $\mathrm{I}, 8$ & $\mathrm{I}, 8$ \\
\hline Zigomático & 4,0 & 3,2 \\
\hline Risório & 3,3 & 2,8 \\
\hline Orbicular da boca & 2,8 & $\mathrm{I}, 3$ \\
\hline
\end{tabular}

Fonte: A autora (2021)

A figura abaixo mostra os pontos de aplicação da toxina botulínica no paciente

Figura 2 - Pontos de aplicação da toxina botulínica no rosto do paciente
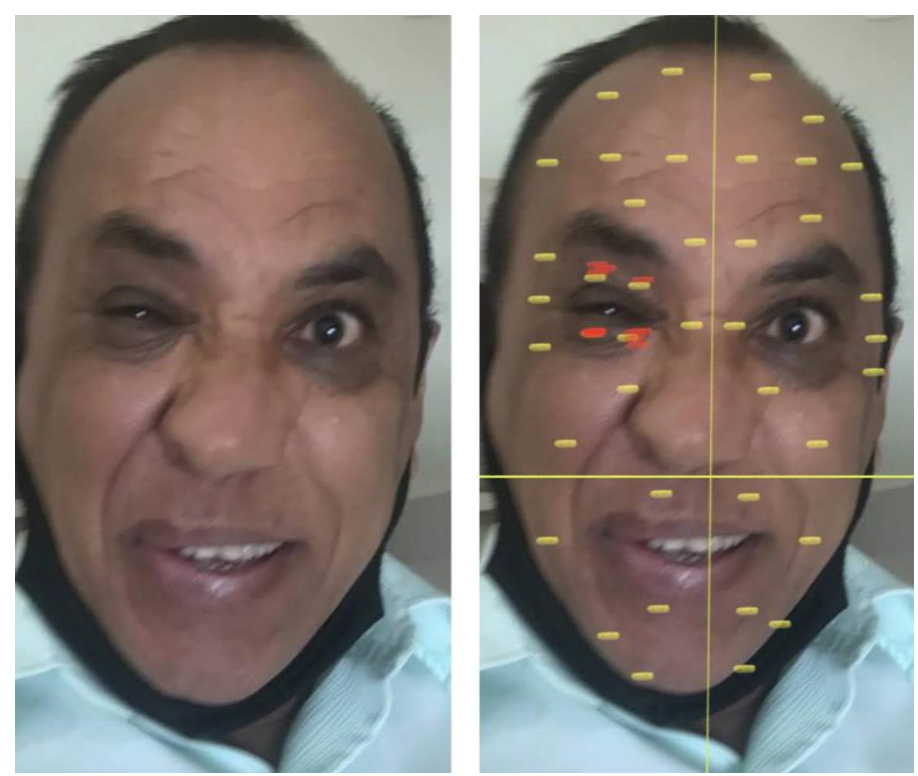

Fonte: A autora (202I)

O tratamento consiste em Io sessões com intervalos de 48 a 72 horas, conforme o Protocolo do aparelho Elite Duo da DMC (2017). Nas primeiras duas sessões as aplicações devem iniciar com uma energia de $\mathrm{IJ}$ a $2 \mathrm{~J}$, por ponto, sendo gradativamente aumentado até chegar a $4 \mathrm{~J}$. A tabela abaixo mostra os protocolos de aplicações para laserterapia com relação aos ramos do nervo facial, tipo de laser, energia e fluência. 
Tabela 2 - Protocolos de aplicação de laserterapia

\begin{tabular}{llcl}
\hline Ramos do nervo facial & Laser & Energia & Fluência \\
\hline Temporal & Infra vermelho & 1 a 4 J & 10 a $100 \mathrm{~J} / \mathrm{cm}^{2}$ \\
Zigomático & Infravermelho & 1 a $4 \mathrm{~J}$ & 10 a $100 \mathrm{~J} / \mathrm{cm}^{2}$ \\
Bucal & Infravermelho & 1 a 4 J & 10 a $100 \mathrm{~J} / \mathrm{cm}^{2}$ \\
Mandibular & Infravermelho & 1 a 4 J & 10 a $100 \mathrm{~J} / \mathrm{cm}^{2}$ \\
Cervical & Infravermelho & 1 a 4 J & 10 a $100 \mathrm{~J} / \mathrm{cm}^{2}$ \\
\hline \hline
\end{tabular}

Fonte: A autora (2021)

A figura abaixo mostra os pontos de aplicação de laserterapia no paciente.

Figura 3 - Pontos de aplicação de laserterapia

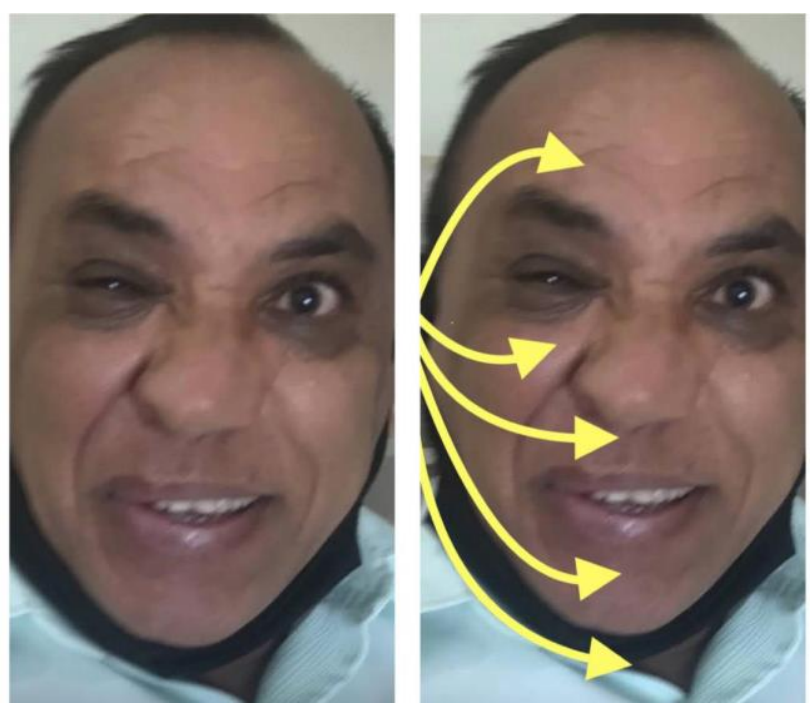

Fonte: A autora (2021)

Após duas semanas de tratamento, foi possível observar uma melhora na fisionomia facial do paciente. Essa mudança pode ser observada na figura abaixo.

Figura 4 - Fisionomia do paciente após duas semanas de tratamento 

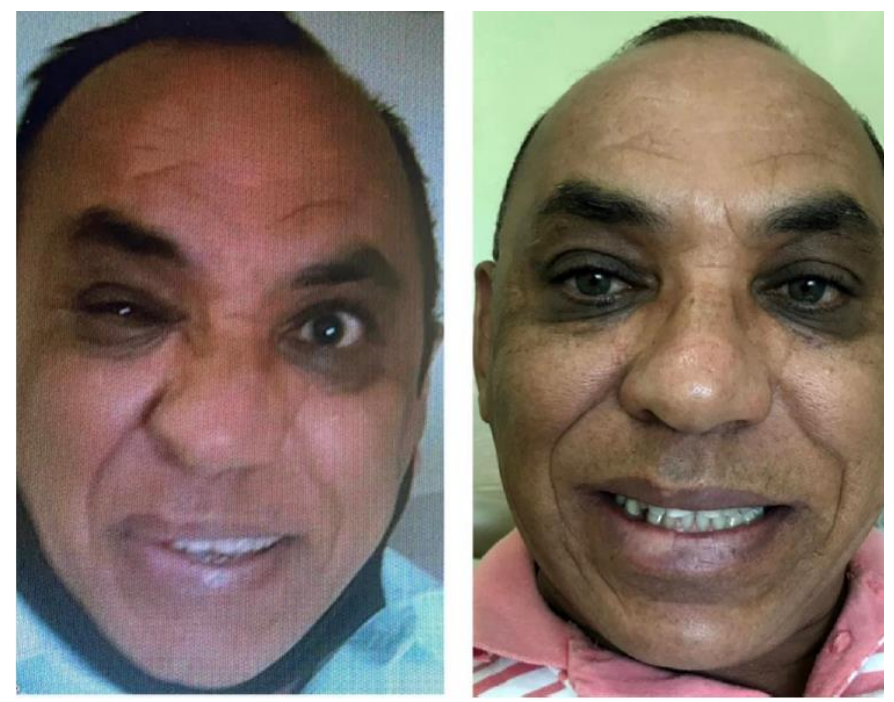

Fonte: A autora (2021)

Para o tratamento, foi realizado um protocolo de duas vezes por semana o tratamento de laserterapia e o uso da vitamina do complexo B diariamente, uma vez ao dia.

Após três meses de tratamento, não houve mais necessidade de nova aplicação de toxina botulínica. Os resultados do tratamento podem ser vistos na figura abaixo.
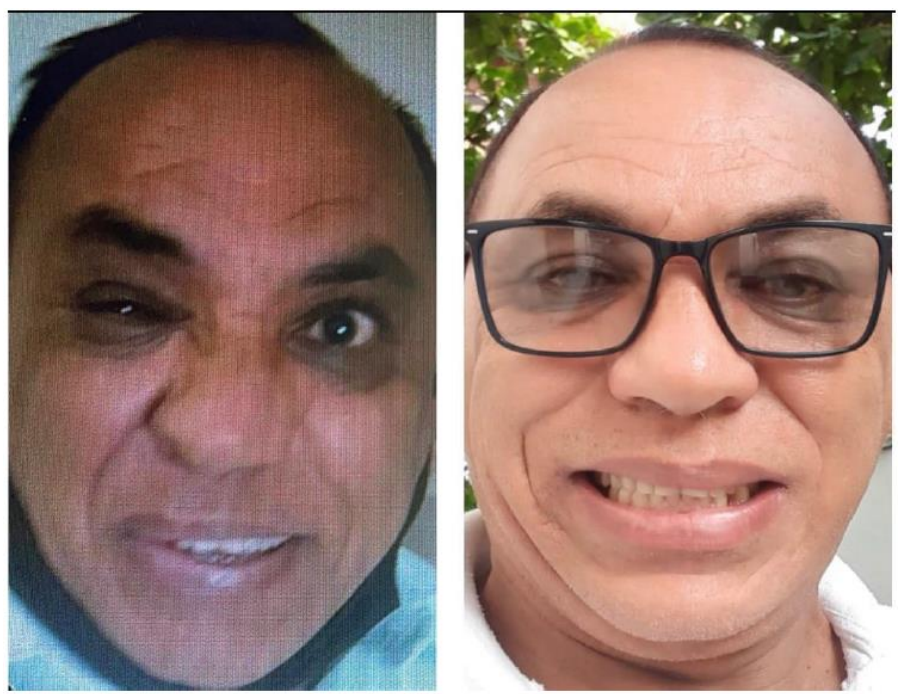

Fonte: A autora (2021)

Após análise das imagens antes e após o tratamento, é possível perceber que todos os objetivos propostos foram atingidos. A conduta terapêutica mostrou-se satisfatória e, 
principalmente, a auto estima do paciente foi recuperada com a melhora do aspecto facial. O acompanhamento do paciente continua semanalmente, sem necessidade de aplicação de Toxina Botulínica até o momento.

\section{DISCUSSÃO}

Segundo Gomez, Vasconcelos e Moraes (1999), a paralisia parcial periférica requer abordagem médica, fisioterapêutica e fonoaudiológica, sendo a fisioterapia um recurso indispensável para reestabelecer o tropismo, a força e a função muscular. Os recursos sugeridos pela literatura são a cinesioterapia, massagem e eletrotermoterapia, confirmados por ensaio clínico aleatório e revisão sistemática.

Viegas, et al (200I) afirma que a laserterapia de baixa potência é uma terapia com fundamento na interação com os tecidos biológicos e cada comprimento de onda irá atingir os efeitos desejados com base na sua absorção através dos cromóforos, que atingem as células onde irá ocorrer a absorção da energia dos fótons emitidos.

\section{CONCLUSÃO}

Os resultados desejados foram alcançados com o tratamento proposto, mesmo com a sequela apresentada pela não abordagem clínica no início dos sintomas.

É importante ressaltar a necessidade de esclarecimento da população em relação ao início imediato do tratamento clínico, visto que a incidência da paralisia facial periférica é relativamente alta dentro dos grupos de risco, amenizando assim as sequelas por tempo prolongado.

Esse protocolo poderá ser utilizado como terapia complementar no tratamento da paralisia de Bell.

Apesar do sucesso do tratamento, ainda é necessário acompanhar a evolução do paciente para afirmar com clareza a reversão definitiva da sequela.

\section{REFERÊNCIAS}

ADONI, T. Paralisia facial periférica. São Paulo - SP, 2008. 
CARVALHO, R. M. L. S.; et al. Tratamento do blefaroespasmo e distonias faciais correlatas com toxina botulínica - estudo de 16 casos. Goiânia - GO, 2002.

DMC IMPORTAÇÃO E EXPORTAÇÃO DE EQUIPAMENTOS. Elite Duo Sistema para Tratamento em Estética: Manual do Usuário. V.4. 2017. Disponível em: $\langle$ http://www.dmcgroup.com.br/arquivos/manual/man_elite_duo_port.pdf〉 Acesso em: 20/04/2021.

DOMINGOS, M. M. O uso da toxina botulínica em doentes com hipercinesia muscular facial contralateral à paralisia facial. São Paulo - SP, 2006.

FALAVIGNA, A.; et al. Paralisia de Bell: fisiopatologia e tratamento. Caxias do Sul - RS, 2008.

GOMEZ, M. V. S. G., VASCONCELOS, L. G. E., MORAES, M. F. B. B. Trabalho miofuncional na paralisia facial. 1999.

MATOS, C.; et al. Paralisia facial periférica: o papel da medicina física e de reabilitação. Lisboa - PT, 201 .

OLIVEIRA, F. A. Efeitos neurodegenerativos causados pela deficiência de vitamina B. Belo Horizonte - MG, 2006.

TESSITORE, A.; PFELSTICKER, L. N.; PASCHOAL, J. R. Aspectos neurofisiológicos da musculatura facial visando a reabilitação na paralisia facial. São Paulo - SP.

RENATA, E.; AMADO, E. T.; OTA, C. C. C. Mecanismo de ação de injetáveis utilizados na biomedicina estética. Curitiba - PR, 2015. 
REVISTA BRASILEIRA DE CIRURGIA PLÁSTICA. Protocolo de aplicação bilateral de toxina botulínica tipo A para evitar assimetria no tratamento de espasmo hemifacial. São Paulo - SP, 2015.

ROBERTO, T. S.; MAGNONI, D.; CUKIER, C. Aplicações clínicas das vitaminas do complexo B. São Paulo - SP.

RODRIGUES, O. B. Regeneração axonal durante administração sistêmica de tiamina, peridoxina e cianocobalamina. Piracicaba - SP, 2003.

VALENÇA, M. M.; et al. Laserterapia associada ao tratamento da paralisia facial de Bell. 2006.

VIEGAS, V. N.; et al. Paralisia facial periférica idiopática de Bell. São Paulo - SP, 200 .

YOSHIMOTO, M.; SALLES, M. B. Avaliação histórica da regeneração nervosa. São Paulo - SP, 2010.

ZANON, R. G.; et al. Expressão do complexo de histocompatibilidade principal de classe I (MHC I) no sistema nervoso central: plasticidade sináptica e regeneração. CAMPINAS SP, 2010. 